\title{
The energy equality for weak solutions to the equations of non-Newtonian fluids
}

\section{Jiaqi Yang}

Key Laboratory for Mechanics in Fluid Solid Coupling Systems, Institute of Mechanics, Chinese Academy of Sciences, Beijing 100190, China

\section{A R T I C L E I N F O}

\section{Article history:}

Received 17 July 2018

Received in revised form 12 September 2018

Accepted 12 September 2018

Available online $\mathrm{xxxx}$

\section{Keywords:}

Non-Newtonian fluids

Weak solution

Energy equality

\begin{abstract}
A B S T R A C T
In this short paper, we extend the result of Shinbrot (1974) to an incompressible fluid with shear dependent viscosity. It is shown that a weak solution to the equations of non-Newtonian fluids lying in a space $L^{q}\left(0, T ; L^{p}\right)$ satisfies an energy equality, where $\frac{2 r}{r-1} \leq p \leq \frac{2 r}{r-2}$ and $\frac{1}{p}+\frac{1}{q} \leq \frac{r-1}{r}$, if $r>2 ; p \geq \frac{2 r}{r-1}$ and $\frac{r-1}{p}+\frac{1}{q}=\frac{r-1}{2}$, if $\frac{2(n+1)}{n+2}<r \leq 2$. In particular, our result implies that the weak solution must satisfy the energy equality when $r \geq \frac{3 n+2}{n+2}$, which is consistent with the known fact.
\end{abstract}

(C) 2018 Elsevier Ltd. All rights reserved.

\section{Introduction}

Let $\Omega \subset \mathbb{R}^{n}(n \geq 2)$ be a domain with a sufficiently smooth boundary. We consider a non-Newtonian incompressible fluid which is governed by the following system

$$
\begin{cases}\mathbf{u}_{t}+\mathbf{u} \cdot \nabla \mathbf{u}-\operatorname{div}\left(|D(\mathbf{u})|^{r-2} D(\mathbf{u})\right)+\nabla \pi=0, & \text { in } \Omega \times(0, T), \\ \operatorname{div} \mathbf{u}=0, & \text { in } \Omega \times(0, T), \\ \mathbf{u}(0, x)=\mathbf{u}_{0}(x), & \text { in } \Omega .\end{cases}
$$

where $\mathbf{u}=\left(u_{1}, u_{2}, u_{3}\right)$ denotes the unknown velocity of the fluid and $\pi$ the pressure, and

$$
D(\mathbf{u})=\frac{1}{2}\left(\nabla \mathbf{u}+(\nabla \mathbf{u})^{T}\right) .
$$

We first give the definition of a weak solution to (1). To this end, we denote by $C_{0}^{\infty}(\Omega)$ the space of smooth functions with compact support. Let $C_{0, \sigma}^{\infty}(\Omega)=\left\{\boldsymbol{\varphi} \in C_{0}^{\infty}(\Omega) \mid \nabla \cdot \varphi=0\right\} \cdot L_{\sigma}^{p}\left(\mathbb{R}^{3}\right)$ denotes the closure of $C_{0, \sigma}^{\infty}(\Omega)$ in the norm $\|\cdot\|_{p} . \dot{H}_{0, \sigma}^{1, p}(\Omega)$ denotes the closure of $C_{0, \sigma}^{\infty}(\Omega)$ in the norm $\|\nabla \cdot\|_{p}$. We write

$$
\|\mathbf{u}\|_{p, q}=\left(\int_{0}^{T}\|\mathbf{u}(t)\|_{p}^{q} d t\right)^{\frac{1}{q}}, \quad 1 \leq q<\infty,
$$

E-mail address: yjq@imech.ac.cn. 
and

$$
\|\mathbf{u}(t)\|_{p, \infty}=e s s \sup _{0<t<T}\|\mathbf{u}\|_{p}
$$

Definition 1. Let $r \geq \frac{2 n}{n+2}, \mathbf{u}_{0} \in L_{\sigma}^{2}(\Omega)$. A vector-valued function $\mathbf{u} \in L^{\infty}\left(0, T ; L_{\sigma}^{2}(\Omega)\right) \cap L^{2}\left(0, T ; \dot{H}_{0, \sigma}^{1, r}(\Omega)\right)$ is called a weak solution to (1) if the following identity

$$
\left(\mathbf{u}_{0}, \boldsymbol{\varphi}(0)\right)+\int_{0}^{T}\left[\left(\mathbf{u}, \partial_{t} \boldsymbol{\varphi}\right)-(\mathbf{u} \cdot \nabla \mathbf{u}, \boldsymbol{\varphi})-\left(|D(\mathbf{u})|^{r-2} D(\mathbf{u}), D(\boldsymbol{\varphi})\right)\right] d t=0
$$

holds for all $\varphi \in C_{0}^{\infty}\left([0, T) ; C_{0, \sigma}^{\infty}(\Omega)\right)$. Here, one restricts $r \geq \frac{2 n}{n+2}$ to make sure that the expression $\int_{0}^{T}(\mathbf{u} \cdot \nabla \mathbf{u}, \boldsymbol{\varphi}) d t$ makes sense.

The existence of weak solutions of (1) is shown in [1,2] with the periodic boundary condition, and in [3] for the whole space. In [4], J. Wolf showed the existence of weak solutions with Dirichlet boundary condition for $r>\frac{2(n+1)}{n+2}$. Moreover, we know that a weak solution satisfies the global energy inequality

$$
\|\mathbf{u}(t)\|_{2}^{2}+2 \int_{0}^{t}\|D(\mathbf{u})(\tau)\|_{r}^{r} d \tau \leq\left\|\mathbf{u}_{0}\right\|_{2}^{2}, \quad t \geq 0 .
$$

A natural question is to consider the possible validity of the energy equality. For Newtonian fluids, i.e. $r=2$, the pioneering results by Prodi [5] and Lions [6] concern the validity of energy equality for a weak solution such that

$$
\mathbf{u} \in L^{4}\left(0, T, L^{4}(\Omega)\right) .
$$

Later, Shinbrot [7] enlarged the range of exponents proving that if a weak solution belongs to

$$
\mathbf{u} \in L^{q}\left(0, T ; L^{p}(\Omega)\right), \quad \frac{1}{p}+\frac{1}{q} \leq \frac{1}{2}, \quad p \geq 4 .
$$

More results in this respect, the reader can see [8] and references therein. However, as far as I know, there is no result for the non-Newtonian fluids. In this paper, we will extend Shinbrot's result to the non-Newtonian fluids. Our main result is stated as follows.

Theorem 1. Let $r>\frac{2(n+1)}{n+2}, r^{\prime}$ is the Hölder conjugate of $r, \mathbf{u}_{0} \in L_{\sigma}^{2}(\Omega)$, and let $\mathbf{u}$ be a weak solution of (1). If $\mathbf{u} \in L^{q}\left(0, T ; L^{p}(\Omega)\right)$, where $2 r^{\prime} \leq p \leq \frac{2 r}{r-2}$ and $\frac{1}{p}+\frac{1}{q} \leq \frac{1}{r^{\prime}}$, if $r>2 ; p \geq 2 r^{\prime}$ and $\frac{r-1}{p}+\frac{1}{q} \leq \frac{r-1}{2}$, if $r \leq 2$. Then $\mathbf{u}$ satisfies the energy equality

$$
\|\mathbf{u}(t)\|_{2}^{2}+2 \int_{0}^{t}\|D(\mathbf{u})(t)\|_{r}^{r} d \tau=\left\|\mathbf{u}_{0}\right\|_{2}^{2}, \quad 0 \leq t<T .
$$

Remark 1. Here, since the existence of weak solutions to (1) is still unsolved for $r \leq \frac{2(n+1)}{n+2}$, see [4], we have to restrict $r>\frac{2(n+1)}{n+2}$.

Remark 2. It is well known that the weak solution is strong and unique for $r \geq \frac{3 n+2}{n+2}$, see for example [2], thus the weak solution satisfies automatically the energy equality. It is remarkable that our result is consistent with this fact. Actually, by virtue of Gagliardo-Nirenberg inequalities and Korn's inequality, one easily verifies

$$
L^{\infty}\left(0, T ; L_{\sigma}^{2}(\Omega)\right) \cap L^{2}\left(0, T ; \dot{H}_{0, \sigma}^{1, r}(\Omega)\right) \hookrightarrow L^{\frac{(n+2) r^{2}-2 n r}{n}}\left(0, T ; L_{\sigma}^{2 r^{\prime}}(\Omega)\right) .
$$

When $r \geq \frac{3 n+2}{n+2}$, one can easily check that

$$
\frac{1}{2 r^{\prime}}+\frac{n}{(n+2) r^{2}-2 n r} \leq \frac{1}{r^{\prime}},
$$

which implies a weak solution of (1) must satisfy the energy equality for any $r \geq \frac{3 n+2}{n+2}$. 


\section{The proof of Theorem 1}

Firstly, we have the following property of weak solutions.

Lemma 2. Let $r>\frac{2(n+1)}{n+2}, \mathbf{u}_{0} \in L_{\sigma}^{2}(\Omega)$, and let $\mathbf{u}$ be a weak solution of $(1)$. Then, after suitable redefinition of $\mathbf{u}$ on a set of values of $t$ of one-dimensional measure zero, we have

$$
\begin{aligned}
(\mathbf{u}(t), \boldsymbol{\varphi}(t))= & \left(\mathbf{u}_{0}, \boldsymbol{\varphi}(0)\right) \\
& +\int_{0}^{t}\left[\left(\mathbf{u}, \partial_{\tau} \boldsymbol{\varphi}\right)-(\mathbf{u} \cdot \nabla \mathbf{u}, \boldsymbol{\varphi})-\left(|D(\mathbf{u})(\tau)|^{r-2} D(\mathbf{u})(\tau), D(\boldsymbol{\varphi})(\tau)\right)\right] d \tau=0
\end{aligned}
$$

holds for all $\varphi \in C_{0}^{\infty}\left([0, T) ; C_{0, \sigma}^{\infty}(\Omega)\right)$ and all $0 \leq t<T$.

This lemma is completely similar with that of Lemma 2.1 in [9], see also [5,10,11], we omit the details here.

Lemma 3. Let $r>1, \phi \in L^{p}(\Omega), \boldsymbol{\psi} \in \dot{H}_{0}^{1, r}(\Omega), \chi \in L^{2}(\Omega) \cap L^{p}(\Omega)$, where

$$
\begin{aligned}
2 r^{\prime} \leq p \leq \frac{2 r}{r-2}, & \text { if } r>2 ; \\
p \geq 2 r^{\prime}, & \text { if } 1<r \leq 2 .
\end{aligned}
$$

Then

$$
|(\phi \cdot \nabla \boldsymbol{\psi}, \chi)| \leq\|\phi\|_{p}\|\nabla \boldsymbol{\psi}\|_{r}\|\chi\|_{2}^{\theta}\|\chi\|_{p}^{1-\theta}
$$

where

$$
\theta=\frac{\frac{1}{q}-\frac{1}{p}}{\frac{1}{2}-\frac{1}{p}}=\frac{2(p-q)}{q(p-2)}
$$

and $q$ is defined by $\frac{1}{p}+\frac{1}{q}=\frac{1}{r^{\prime}}$.

Proof. If $\chi \in L^{q}(\Omega)$, then

$$
|(\phi \cdot \nabla \boldsymbol{\psi}, \boldsymbol{\chi})| \leq\|\phi\|_{p}\|\nabla \boldsymbol{\psi}\|_{r}\|\chi\|_{q} .
$$

From the assumption (5), one can easily check that $2 \leq q \leq p$. Hence we can use interpolation and write $q$ as $\frac{1}{q}=\frac{\theta}{2}+\frac{1-\theta}{p}$, which give us $\theta:=\frac{\frac{1}{q}-\frac{1}{p}}{\frac{1}{2}-\frac{1}{p}}=\frac{2(p-q)}{q(p-2)}$. Thus,

$$
|(\phi \cdot \nabla \boldsymbol{\psi}, \chi)| \leq\|\phi\|_{p}\|\nabla \boldsymbol{\psi}\|_{r}\|\chi\|_{2}^{\theta}\|\chi\|_{p}^{1-\theta} \text {. }
$$

Lemma 4. Let $r>1$, and

$$
\phi \in L^{\tilde{q}}\left(0, T ; L^{p}(\Omega)\right), \boldsymbol{\psi} \in L^{r}\left(0, T ; \dot{H}_{0}^{1, r}(\Omega)\right), \quad \chi \in L^{\infty}\left(0, T ; L^{2}(\Omega)\right) \cap L^{\tilde{q}}\left(0, T ; L^{p}(\Omega)\right),
$$

where $p$ satisfies the assumption (5). Then

(i) If $r>2$ and $\frac{1}{p}+\frac{1}{\tilde{q}}=\frac{1}{r^{\prime}}$, one has

$$
\left|\int_{0}^{T}(\phi(t) \cdot \nabla \boldsymbol{\psi}(t), \chi(t)) d t\right| \leq T^{\left(\frac{1}{r^{\prime}}-\frac{1}{2}\right) \theta}\|\phi\|_{p, \tilde{q}}\|\nabla \boldsymbol{\psi}\|_{r, r}\|\chi\|_{2, \infty}^{\theta}\|\chi\|_{p, \tilde{q}}^{\frac{q}{2}-1},
$$

(ii) If $1<r<2$ and $\frac{r-1}{p}+\frac{1}{\tilde{q}}=\frac{r-1}{2}$, one has

$$
\left|\int_{0}^{T}(\phi(t) \cdot \nabla \boldsymbol{\psi}(t), \boldsymbol{\chi}(t)) d t\right| \leq\|\phi\|_{p, \tilde{q}}\|\nabla \boldsymbol{\psi}\|_{r, r}\|\chi\|_{2, \infty}^{\theta}\|\chi\|_{p, \tilde{q}}^{\frac{q}{2}-1} .
$$


Proof. If $r>2$ and $\frac{1}{p}+\frac{1}{\tilde{q}}=\frac{1}{r^{\prime}}$, one can easily check that

$$
\frac{1}{\tilde{q}}+\frac{1}{r}+\frac{1-\theta}{\tilde{q}}+\left(\frac{1}{r^{\prime}}-\frac{1}{2}\right) \theta=1
$$

then by Hölder's inequality, one can obtain (7) by Lemma 4 . If $1<r<2$ and $\frac{1}{\tilde{q}}+\frac{r-1}{p}=\frac{r-1}{2}$, it is easy to know that

$$
\frac{1}{\tilde{q}}+\frac{1}{r}+\frac{1-\theta}{\tilde{q}}=1
$$

thus, one can get (8) by virtue of Lemma 4 .

Now, using the above result, we can prove Theorem 1.

Proof of Theorem 1. The proof follows [7]. Let

$$
\left(k_{\varepsilon} * \phi\right)(t)=\int_{0}^{t_{0}} k_{\varepsilon}(t-\tau) \phi(\tau) d \tau
$$

be a mollifier, so that $k_{\varepsilon}$ is $C^{\infty}$, real-valued, nonnegative function, supported in $[-\varepsilon, \varepsilon]$, and integrating to unity. Let $\left\{\mathbf{u}_{m}\right\} \subset C_{0}^{\infty}\left([0, \infty) ; C_{0, \sigma}^{\infty}(\Omega)\right)$ be a sequence converging to $\mathbf{u}$ in $L^{2}\left(0, T ; L_{\sigma}^{2}(\Omega)\right) \cap$ $L^{r}\left(0, T ; \dot{H}_{0, \sigma}^{1, r}(\Omega)\right) \cap L^{q}\left(0, T ; L^{p}(\Omega)\right)$. Set $t=t_{0}$ and $\boldsymbol{\varphi}=k_{\varepsilon} * \mathbf{u}_{m}$ in (4). One obtains

$$
\begin{aligned}
& \int_{0}^{t_{0}} k_{\varepsilon}\left(t_{0}-t\right)\left(\mathbf{u}\left(t_{0}\right), \mathbf{u}_{m}(t)\right) d t \\
= & \int_{0}^{t_{0}} k_{\varepsilon}(-t)\left(\mathbf{u}_{0}, \mathbf{u}_{m}(t)\right)+\int_{0}^{t_{0}} \int_{0}^{t_{0}} \partial_{t} k_{\varepsilon}(t-\tau)\left(\mathbf{u}(t), \mathbf{u}_{m}(\tau)\right) d \tau d t \\
& -\int_{0}^{t_{0}} \int_{0}^{t_{0}} k_{\varepsilon}(t-\tau)\left[\left(\mathbf{u}(t) \cdot \nabla \mathbf{u}(t), \mathbf{u}_{m}(\tau)\right)+\left(|D(\mathbf{u})(t)|^{r-2} D(\mathbf{u})(t), D\left(\mathbf{u}_{m}\right)(\tau)\right)\right] d \tau d t .
\end{aligned}
$$

From Lemma 3, we have

$$
\begin{aligned}
& \left|\int_{0}^{t_{0}} \int_{0}^{t_{0}} k_{\varepsilon}(t-\tau)\left(\mathbf{u}(t) \cdot \nabla \mathbf{u}(t), \mathbf{u}_{m}(\tau)-\mathbf{u}(\tau)\right) d \tau d t\right| \\
\leq & \int_{0}^{t_{0}}\|\mathbf{u}(t)\|_{p}\|\nabla \mathbf{u}(t)\|_{r} d t \int_{0}^{t_{0}}\left\|\mathbf{u}_{m}(\tau)-\mathbf{u}(\tau)\right\|_{2}^{\theta}\left\|\mathbf{u}_{m}(\tau)-\mathbf{u}(\tau)\right\|_{p}^{1-\theta} d \tau
\end{aligned}
$$

Since $r^{\prime} \leq q$, we have

$$
\int_{0}^{t_{0}}\|\mathbf{u}(t)\|_{p}\|\nabla \mathbf{u}(t)\|_{r} d t \leq C\left(t_{0}\right)\|\mathbf{u}\|_{p, q}\|\nabla u\|_{r, r} .
$$

On the other hand, since $\frac{1}{2}+\frac{1}{q} \leq 1$, we have

$$
\int_{0}^{t_{0}}\left\|\mathbf{u}_{m}(\tau)-\mathbf{u}(\tau)\right\|_{2}^{\theta}\left\|\mathbf{u}_{m}(\tau)-\mathbf{u}(\tau)\right\|_{p}^{1-\theta} d \tau \leq C\left(t_{0}\right)\left\|\mathbf{u}_{m}-\mathbf{u}\right\|_{2,2}^{\theta}\left\|\mathbf{u}_{m}-\mathbf{u}\right\|_{p, q}^{1-\theta} \rightarrow 0, \quad \text { as } m \rightarrow \infty .
$$

Thus, let $m \rightarrow \infty$ in (9), we have

$$
\begin{aligned}
& \int_{0}^{t_{0}} k_{\varepsilon}\left(t_{0}-t\right)\left(\mathbf{u}\left(t_{0}\right), \mathbf{u}(t)\right) d t \\
= & \int_{0}^{t_{0}} k_{\varepsilon}(-t)\left(\mathbf{u}_{0}, \mathbf{u}(t)\right)+\int_{0}^{t_{0}} \int_{0}^{t_{0}} \partial_{t} k_{\varepsilon}(t-\tau)(\mathbf{u}(t), \mathbf{u}(\tau)) d \tau d t \\
& -\int_{0}^{t_{0}} \int_{0}^{t_{0}} k_{\varepsilon}(t-\tau)\left[(\mathbf{u}(t) \cdot \nabla \mathbf{u}(t), \mathbf{u}(\tau))+\left(|D(\mathbf{u})(t)|^{r-2} D(\mathbf{u})(t), D(\mathbf{u})(\tau)\right)\right] d \tau d t .
\end{aligned}
$$


The term here involving the derivative vanishes if $k$ is chosen to be even. We send $\varepsilon$ to zero in the remaining terms. Because of the usual properties of mollifiers,

$$
\int_{0}^{t_{0}} \int_{0}^{t_{0}} k_{\varepsilon}(t-\tau)\left(|D(\mathbf{u})(t)|^{r-2} D(\mathbf{u})(t), D(\mathbf{u})(\tau)\right) d \tau d t \rightarrow \int_{0}^{t_{0}}\|D(\mathbf{u})(t)\|_{p}^{p} d t .
$$

In addition,

$$
\int_{0}^{t_{0}} k_{\varepsilon}\left(t_{0}-t\right)\left(\mathbf{u}\left(t_{0}\right), \mathbf{u}(t)\right) d t=\int_{0}^{\varepsilon} k_{\varepsilon}(t)\left(\mathbf{u}\left(t_{0}\right), \mathbf{u}\left(t_{0}-t\right)\right) d t
$$

since as a function of $t, \mathbf{u}$ is continuous in the weak topology of $L_{\sigma}^{2}(\Omega)$ (see [4] Theorem 1.3 for example), we have

$$
\int_{0}^{t_{0}} k_{\varepsilon}\left(t_{0}-t\right)\left(\mathbf{u}\left(t_{0}\right), \mathbf{u}(t)\right) d t=\int_{0}^{\varepsilon} k_{\varepsilon}(t)\left[\left\|\mathbf{u}\left(t_{0}\right)\right\|_{2}^{2}+o(1)\right] d t \rightarrow \frac{1}{2}\left\|\mathbf{u}\left(t_{0}\right)\right\|_{2}^{2} .
$$

Similarly,

$$
\int_{0}^{t_{0}} k_{\varepsilon}(-t)\left(\mathbf{u}_{0}, \mathbf{u}(t)\right) d t \rightarrow \frac{1}{2}\left\|\mathbf{u}_{0}\right\|_{2}^{2}
$$

Finally, we consider the nonlinear term in (10). We have

$$
\begin{aligned}
& \int_{0}^{t_{0}} \int_{0}^{t_{0}} k_{\varepsilon}(t-\tau)(\mathbf{u}(t) \cdot \nabla \mathbf{u}(t), \mathbf{u}(\tau)) d \tau d t-\int_{0}^{t_{0}}(\mathbf{u}(t) \nabla \mathbf{u}(t), \mathbf{u}(t)) d t \\
= & \int_{0}^{t_{0}}\left(\mathbf{u}(t) \cdot \nabla \mathbf{u}(t),\left(k_{\varepsilon} * \mathbf{u}\right)(t)-\mathbf{u}(t)\right) d t .
\end{aligned}
$$

By Lemma 4, this is bounded by

$$
C\left(t_{0}\right)\|\mathbf{u}\|_{p, q}\|\nabla \mathbf{u}\|_{r, r}\left\|k_{\varepsilon} * \mathbf{u}-\mathbf{u}\right\|_{2, \infty}^{\theta}\left\|k_{\varepsilon} * \mathbf{u}-\mathbf{u}\right\|_{p, q}^{\frac{q}{2}-1} .
$$

This goes to zero because of usual properties of mollifiers. Thus (11) goes to zero. Now, we prove $\int_{0}^{t_{0}}(\mathbf{u}(t) \cdot \nabla \mathbf{u}(t), \mathbf{u}(t))=0$. From Lemma 4 , we have that the function $F$ defined by

$$
F(\boldsymbol{\psi}, \boldsymbol{\chi})=\int_{0}^{t_{0}}(\mathbf{u}(t) \cdot \nabla \boldsymbol{\psi}(t), \chi(t)) d t
$$

is continuous on $L^{2}\left(0, t_{0} ; \dot{H}_{0}^{1, r}(\Omega)\right) \times L^{q}\left(0, t_{0} ; L^{p}(\Omega)\right)$. On the other hand, integration by parts shows that $F(\boldsymbol{\psi}, \boldsymbol{\psi})=0$ if $\boldsymbol{\psi}$ is smooth. Let $\left\{\mathbf{u}_{m}\right\}$ be a sequence from $C_{0}^{\infty}\left([0, T) ; C_{0, \sigma}^{\infty}(\Omega)\right)$ converging to $\mathbf{u}$ in the appropriate spaces. Then we find

$$
0=F\left(\mathbf{u}_{m}, \mathbf{u}_{m}\right) \rightarrow F(\mathbf{u}, \mathbf{u})
$$

All of this shows that

$$
\int_{0}^{t_{0}} \int_{0}^{t_{0}} k_{\varepsilon}(t-\tau)(\mathbf{u}(t) \cdot \nabla \mathbf{u}(t), \mathbf{u}(\tau)) d \tau d t \rightarrow 0
$$

as $\varepsilon \rightarrow 0$. Now, let $\varepsilon \rightarrow 0$ in (9), we have

$$
\frac{1}{2}\left\|\mathbf{u}\left(t_{0}\right)\right\|_{2}^{2}+\int_{0}^{t}\|D(\mathbf{u})(t)\|_{r}^{r} d \tau=\left\|\mathbf{u}_{0}\right\|_{2}^{2},
$$

which is (3) for $t=t_{0}$. Since $t_{0}$ is arbitrary, we have finished the proof of Theorem 1.

\section{Acknowledgment}

I thank the anonymous referee for giving me many helpful suggestions and comments which led to improvement of the presentation. 


\section{References}

[1] O.A. Ladyzhenskaya, The Mathematical Theory of Viscous Incompressible Flow, second ed., Gordon and Breach, New York, 1969.

[2] J. Málek, J. Nečas, M. Rokyta, M. Ružička, Weak and Measure-valued Solutions to Evolutionary PDEs, 1996s.

[3] M. Pokorny, Cauchy problem for the non-newtonian viscous incompressible fluid, Appl. Math. 41 (3) (1996) $169-201$.

[4] J. Wolf, Existence of weak solutions to the equations of non-stationary motion of non-Newtonian fluids with shear rate dependent viscosity, J. Math. Fluid Mech. 9 (1) (2007) 104-138.

[5] G. Prodi, Un teorema di unicità per le equazioni di Navier-Stokes, Ann. Mat. Pura Appl. 48 (1) (1959) $173-182$.

[6] J.L. Lions, Sur la régularité et l'unicité des solutions turbulentes des équations de Navier Stokes, Rend. Semin. Mat. Univ. Padova 30 (1960) 16-23.

[7] M. Shinbrot, The energy equation for the Navier-Stokes system, SIAM J. Math. Anal. 5 (6) (1974) $948-954$.

[8] L.C. Berselli, E. Chiodaroli, Remarks on the energy equality for weak solutions to Navier-Stokes equations, 2018, arXiv preprint arXiv:1807.02667 [math. AP].

[9] G.P. Galdi, An introduction to the Navier-Stokes initial-boundary value problem, in: Fundamental Directions in Mathematical Fluid Mechanics, in: Adv. Math. Fluid Mech., Birkhäuser, Basel, 2000, pp. 1-70.

[10] E. Hopf, Über die Anfangswertaufgabe für die hydrodynamischen Grundgleichungen, Math. Nachr. 4 (1951) $213-231$.

[11] J. Serrin, The initial value problem for the Navier-Stokes equations, in: Rudolph E. Langer (Ed.), Nonlinear Problems, University of Wisconsin Press, Madison, 1963, pp. 69-98. 